\title{
Simple one-week method to construct gene- targeting vectors: application to production of human knockout cell lines
}

\author{
Susumu liizumi ${ }^{1}$, Yuji Nomura ${ }^{1}$, Sairei So ${ }^{1}$, Koichi Uegaki ${ }^{1}$, Kayoko Aoki¹, \\ Kei-ichi Shibahara ${ }^{2}$, Noritaka Adachi ${ }^{1}$, and Hideki Koyama ${ }^{1}$ \\ ${ }^{1}$ Yokohama City University, Yokohama and 2National Institute of Genetics, Mishima, \\ Japan
}

BioTechniques 41:311-316 (September 2006)

doi 10.2144/000112233

Targeted gene disruption is a powerful tool for studying gene function in cells and animals. In addition, this technology includes a potential to correct disease-causing mutations. However, constructing targeting vectors is a laborious step in the gene-targeting strategy, even apart from the low efficiency of homologous recombination in mammals. Here, we introduce a quick and simplified method to construct targeting vectors. This method is based on the commercially available MultiSite Gateway ${ }^{\circledR}$ technology. The sole critical step is to design primers to PCR amplify genomic fragments for homologous DNA arms, after which neither ligation reaction nor extensive restriction mapping is necessary at all. The method therefore is readily applicable to embryonic stem (ES) cell studies as well as all organisms whose genome has been sequenced. Recently, we and others have shown that the human pre$B$ cell line Nalm-6 allows for high-efficiency gene targeting. The combination of the simplified vector construction system and the high-efficiency gene targeting in the Nalm-6 cell line has enabled rapid disruption of virtually any locus of the human genome within one month, and homozygous knockout clones lacking a human gene of interest can be created within 2-3 months. Thus, our system greatly facilitates reverse genetic studies of mammalian-particularly human-genes.

\section{INTRODUCTION}

Targeted gene disruption provides a powerful means for studying gene function by a reverse genetic approach $(1,2)$. This technology depends on homologous recombination reactions that occur, albeit rarely, between transfected DNA (i.e., targeting vector) and the host genome. In mice, a large number of genes have been knocked out thus far using embryonic stem (ES) cells, and their physiological functions have been elucidated. More recently, reverse genetic studies using chicken DT40 cells have made a significant contribution to our understanding of cellular functions of a variety of genes (3). It is important to note, however, that the findings in model organisms are not always the case in human cells. Hence, reverse genetic analysis using human somatic cells would be of greater importance in the postgenome era when one attempts to reliably analyze the function of human genes. In human cultured cells, however, the frequency of gene targeting is typically too low for these approaches to be feasible $(2,4)$. In this regard, it is noteworthy that we and others have shown that Nalm-6, a human pre-B acute lymphoblastic leukemia (ALL) cell line, is highly proficient in genetargeting experiments (5-8).

A rate-limiting step in genetargeting experiments, apart from the low efficiency of homologous recombination, is constructing targeting vectors. Vector construction typically involves cloning and mapping of genomic DNA fragments, which is highly time-consuming. Fortunately, the completion of the human genome sequence has greatly simplified those tedious processes, since virtually all DNA fragments can be readily PCRamplified with specific primers (which must be designed not to include repetitive sequences such as Alu, however).
Nevertheless, the subsequent stepnamely assembly of a genomic DNA fragment(s) with selectable markerscan still be a time-consuming and complicated process, partly due to the necessity of searching for appropriate restriction sites for ligation reactions as well as those for vector linearization. Thus, the success of vector construction depends crucially on the skill of the researcher. In this paper, we report a quick and simplified method for vector construction that we believe is simpler and quicker than any other methods reported thus far. We also show that virtually any genomic locus can be disrupted within one month in the human Nalm- 6 cell line.

\section{MATERIALS AND METHODS}

\section{Vector Construction Using the MultiSite Gateway ${ }^{\circledR}$ System}

To construct the pDEST DTA-MLS plasmid, a diphtheria toxin A (DT-A) gene cassette, which was excised from pMC1DT-ApA (Kurabo, Osaka, Japan) and a 58-mer multiple-linearization site (MLS) fragment carrying PacI, SwaI, I-SceI, AscI, and PmeI sites were sequentially inserted at the $N d e \mathrm{I}$ and Aat II sites of pDEST ${ }^{\mathrm{TM}} \mathrm{R} 4-\mathrm{R} 3$, respectively (Invitrogen, Carlsbad, CA, USA; see Supplementary Figure S1 available online at www.BioTechniques.com). The 58-mer DNA fragment was made by annealing oligonucleotides MLS-A and MLS-B (Table 1).

To create entry clones for floxed positive selectable markers hygromycin-resistance gene $\left(\right.$ Hygr $\left.^{r}\right)$, puromycin-resistance gene (Puror), and histidinol-resistance gene $\left(H i s^{r}\right)$, each marker gene was subcloned into the donor vector pDONR ${ }^{\mathrm{TM}} 221$, as illustrated in Supplementary Figure S2. Briefly, an entry clone plasmid, pENTR loxP, was first created by utilizing a DNA fragment generated by PCR with attB1- and attB2-containing primers (246-B1 and 246-B2, respectively; Table 1) using pBS246 (Gibco ${ }^{\circledR}$; Invitrogen) as template. Floxed marker gene plasmids were constructed as depicted in Supplementary Figure S2, and each marker gene was subcloned into pENTR $\operatorname{loxP}$ at the NotI sites, 
Table 1. Oligonucleotides

\begin{tabular}{|c|c|c|}
\hline Name & Sequence & Use \\
\hline MLS-A & 5'-GTTTAAACGGCGCGCCTAGGGATAACAGGGTAATATTTAAATTTAATTAAACGT-3' & $\begin{array}{l}\text { Annealed to MLS-B to yield the } \\
\text { MLS fragment }{ }^{a}\end{array}$ \\
\hline 246-B1 & 5'-GGGGACAAGTTTGTACAAAAAAGCAGGCTGCGGCCGCACGTCTAAGAAACCATT-3' & $\begin{array}{l}\text { Forward primer with attB1 se- } \\
\text { quence for construction of } \mathrm{pENTR} \\
\text { loxPb }\end{array}$ \\
\hline L4-1 & 5'-GGGGACAACTTTGTATAGAAAAGTTGGTTGCTTGGATCAGCAAGCATTAT-3' & $\begin{array}{l}\text { Forward primer with attB4 se- } \\
\text { quence for } 5^{\prime} \text { arm of } L / G 4\end{array}$ \\
\hline L4-2 & 5'-GGGGACTGCTTTTTTTGTACAAACTTGTCCCTGTCAGACTACCACATAGCA-3' & $\begin{array}{l}\text { Reverse primer with attB1 se- } \\
\text { quence for } 5^{\prime} \text { arm of } L / G 4\end{array}$ \\
\hline L4-4 & 5'-GGGGACAACTTTGTATAATAAAGTTGGCACACATAGTATCGCATGGATCA-3' & $\begin{array}{l}\text { Reverse primer with attB3 se- } \\
\text { quence for } 3^{\prime} \text { arm of } L I G 4 c\end{array}$ \\
\hline L4-N & 5'-TAGCATAGCACTTAATCACCTTCC-3' & $\begin{array}{l}\text { Primer for screening for } L I G 4 \text { tar- } \\
\text { geting events }\end{array}$ \\
\hline $\begin{array}{l}\text { Universal } \\
\text { primer A }\end{array}$ & 5'-AATAATGGTTTCTTAGACGTGCG-3' & $\begin{array}{l}\text { Universal primer for screening tar- } \\
\text { geting events } \mathrm{c}, \mathrm{d}\end{array}$ \\
\hline $\begin{array}{l}\text { Universal } \\
\text { primer B }\end{array}$ & 5'-AGGTTCACTAGTACTGGCCATTG-3' & $\begin{array}{l}\text { Universal primer for screening tar- } \\
\text { geting events } \mathrm{c}, \mathrm{d}\end{array}$ \\
\hline L4-F & 5'-CACTGGAACTCATGGAGATGCTGG-3' & $\begin{array}{l}\text { Forward primer in LIG4 for confir- } \\
\text { mation of LIG4 knockout cellsc }\end{array}$ \\
\hline
\end{tabular}

yielding pENTR lox-Hyg, pENTR loxPuro, and pENTR lox-His.

To generate targeting vectors for the human DNA ligase IV gene (LIG4), 2.2- and 2.0-kb LIG4 genomic fragments were obtained by PCR with ExTaq ${ }^{\mathrm{TM}}$ DNA polymerase (Takara Bio, Otsu, Japan) using Nalm-6 genomic DNA as template and were used as $5^{\prime}$ and $3^{\prime}$ arms, respectively. The primers used were L4-1 and L4-2 for the 5' arm and L4-3 and L4-4 for the 3' arm (see Table 1). By using the MultiSite Gateway system (more specifically, pENTR lox-Hyg, pENTR lox-Puro, and pDEST DTA-MLS), a floxed $H_{y}{ }^{r}$ or Puro ${ }^{r}$ gene was inserted between the $5^{\prime}$ and $3^{\prime}$ arms on a plasmid carrying a DT-A gene, thus yielding targeting vectors pLIG4-Hyg and pLIG4-Puro.
The targeting construct and knockout strategies for the $K U 80$ gene have been described earlier (9).

\section{Cell Culture and Transfection}

Nalm-6 cells were maintained in ES medium (Nissui Seiyaku, Tokyo, Japan) supplemented with $10 \%$ calf serum (Hyclone, Logan, UT, USA) and $50 \mu \mathrm{M} 2$-mercaptoethanol at $37^{\circ} \mathrm{C}$ in a humidified atmosphere of $5 \% \mathrm{CO}_{2}$ in air. DNA transfection was performed as described previously $(6,7,10)$. Briefly, $4 \times 10^{6}$ cells were electroporated with $4 \mu \mathrm{g}$ linearized targeting vector in a 40$\mu \mathrm{L}$ chamber of Electro Gene Transfer Equipment (GTE-1; Shimadzu, Kyoto, Japan). After a 22- to 24-h incubation, the cells were then incubated for
2-3 weeks at $37^{\circ} \mathrm{C}$ in agarose medium containing $0.4 \mathrm{mg} / \mathrm{mL}$ hygromycin $\mathrm{B}$ (Wako Pure Chemical, Osaka, Japan) or $0.5 \mu \mathrm{g} / \mathrm{mL}$ puromycin (Wako). Genomic DNA was isolated from drugresistant colonies and subjected to PCR and Southern blot analyses.

\section{PCR Screening for LIG4 Gene Targeting}

The targeting vector pLIG4-Puro was introduced into Nalm-6 cells, and puromycin-resistant clones were subjected to PCR analysis using a LIG4-specific primer, L4-N, and the universal primer $\mathrm{A}$, which corresponds to nucleotide positions 26-4 of pBS246 (Table 1). Subsequently, pLIG4-Hyg was introduced into $L I G 4+/-$ cells, 


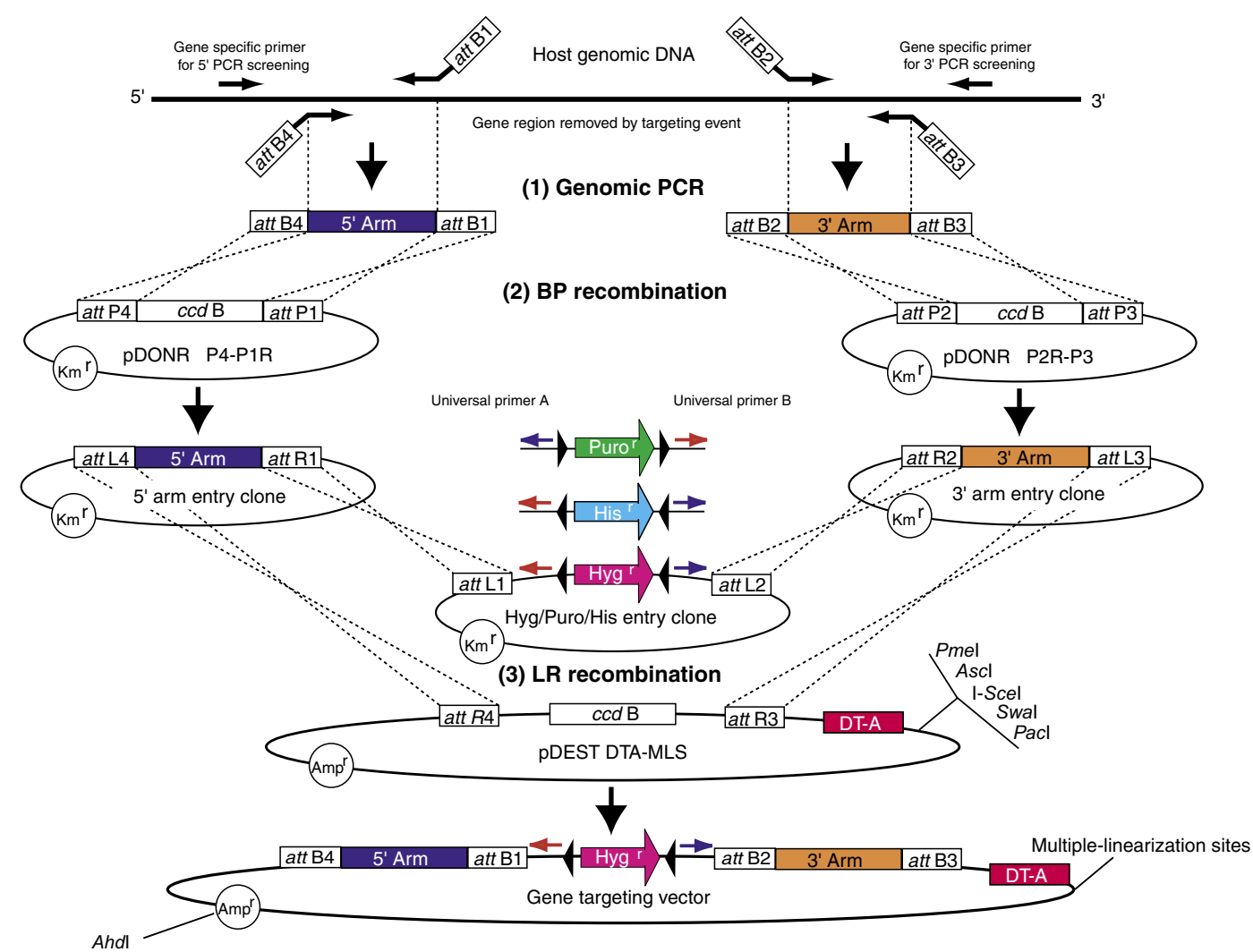

Figure 1. Novel method to construct gene-targeting vectors. The method is based using the MultiSite Gateway system and consists of three steps: (1) genomic PCR with attB-containing primers; (2) BP recombination of each arm fragment; and (3) LR recombination of four DNA fragments. Because neither ligation reaction nor restriction mapping is necessary at all, one can make desired targeting vectors within 5-10 days after primers' arrival. Triangles represent lox $\mathrm{P}$ sequences. Puro $^{r}$, puromycin-resistance gene; His ${ }^{r}$, histidinol-resistance gene; $\mathrm{Hyg}^{r}$, hygromycinresistance gene; $\mathrm{Km}^{r}$, kanamycin-resistance gene; $A m p^{r}$, ampicillin-resistance gene; DT-A, a gene that codes for a diphtheria toxin A fragment.

and hygromycin-resistant clones were subjected to PCR analysis using the L4-N primer and the universal primer $\mathrm{B}$, which corresponds to nucleotide positions 224-246 of pBS246 (Table 1). Two additional primers, L4-F and L4$\mathrm{R}$, which fail to anneal to the disrupted LIG4 locus, were also used for PCR analysis (Table 1). To verify the correct targeting event, PCR-positive clones were subjected to Southern and Western blot analyses $(6,9)$.

\section{RESULTS AND DISCUSSION}

\section{Quick and Simple Method to Construct Targeting Vectors}

As illustrated in Figure 1, the vector construction method is based on the MultiSite Gateway technology, which is commercially available from Invitrogen (MultiSite Gateway Three-Fragment Vector Construction kit). This system allows for one-step assembly of four DNA fragments (the backbone of a destination vector and three insert fragments subcloned as entry clones), by virtue of three independent site-specific recombination (LR recombination) reactions at one time. In order to make the most of this system for targeting-vector construction, we made two major improvements. First, we inserted a DTA gene cassette and an MLS sequence into the destination vector pDEST R4-R3, yielding the plasmid pDEST DTA-MLS (see Supplementary Figure S1). The DT-A gene, which codes for a diphtheria toxin A fragment, serves as a negative selectable marker that allows for selection against unwanted random integrants. The MLS sequence facilitates linearization of targeting vectors, as it contains the 18-bp I-SceI endonuclease cleavage site and restriction sites for four 8-base cutters (PacI, SwaI, $A s c \mathrm{I}$, and PmeI). Second, we prepared a series of floxed positive selectable markers (Hygr, Puro ${ }^{r}$, and His $^{r}$ ), each being subcloned into the donor vector pDONR 221. Accordingly, these marker fragments are all ready to use as entry clones (Supplementary Figure S2). The use of floxed positive selectable markers is of particular importance when one attempts to generate doubleor triple-mutant cells, as a floxed region can easily be removed from the genome by transient expression of Cre recombinase in the cell.

With the plasmid pDEST DTAMLS and the entry clones for positive selectable markers, the remaining steps for vector construction are: $(i)$ isolation of genomic DNA fragments for $5^{\prime}$ and $3^{\prime}$ arms; (ii) subcloning of these arm fragments using BP recombination reactions to create $5^{\prime}$ and $3^{\prime}$ entry clones; and (iii) one-step assembly of four DNA fragments, namely LR recombination reactions between the two entry clones, an entry clone for $\mathrm{Hyg}^{r}$, $\mathrm{Puro}^{r}$, or $\mathrm{His}^{r}$, and the destination vector pDEST DTA-MLS (see Figure 1). The $5^{\prime}$ arm should be PCR-amplified using a forward primer starting with an attB4 sequence and a reverse primer with an att $\mathrm{B} 1$ sequence, and the amplified PCR fragment must be subcloned into the donor vector pDONR P4-P1R. (It should be noted that in the MultiSite Gateway system, each arm fragment must be subcloned as an entry clone that contains an $a t t \mathrm{~L}$ 


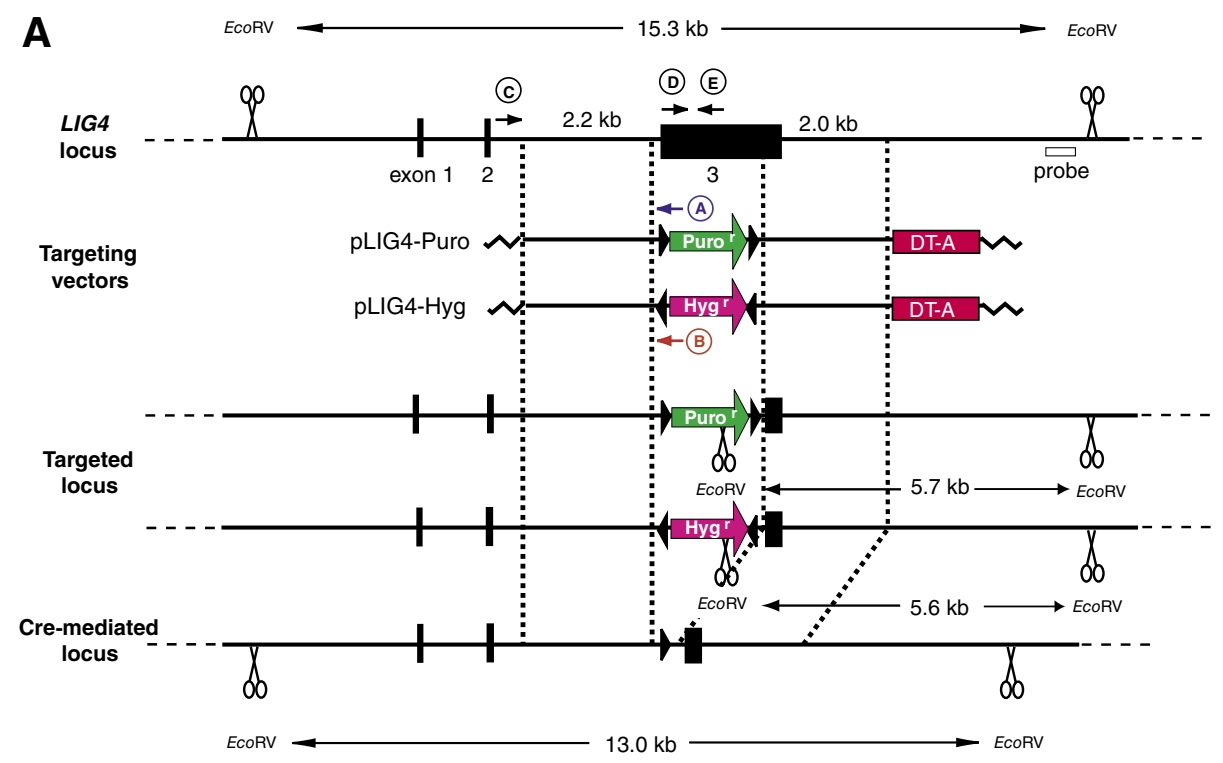

B
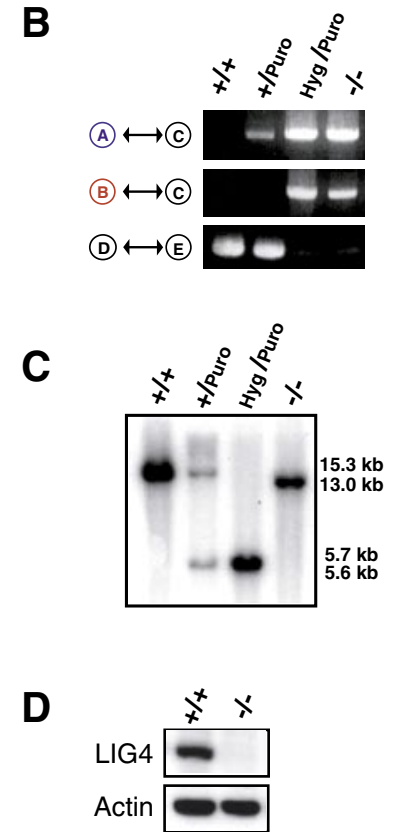

Figure 2. Targeted disruption of the human LIG4 gene. (A) LIG4 disruption in Nalm-6 cells. The human LIG4 gene is composed of three exons, located on chromosome 13q33.3 (genome.ucsc.edu). Arrows A-E stand for PCR primers: A, universal primer A; B, universal primer B; C, L4-N; D, L4-F; and E, L4-R. (B) PCR analysis. +/+, wild-type; +/Puro, LIG4+/-; Hyg/Puro, LIG4 $4^{--} ;-/-, L I G 4^{-/-}$(where selectable markers have been excised). The primers used are shown in panel A. (C) Southern blot analysis of EcoRV-digested genomic DNA. The probe used is shown in panel A. (D) Western blot analysis for LIG4 protein. Samples used contained $20 \mu \mathrm{g}$ total protein extracted from wild-type (+/+) and $L I G 4^{-/-}(-/-)$cells. Actin served as a loading control. Puror , puromycin-resistance gene; $\mathrm{Hyg}^{r}$, hygromycin-resistance gene; DT-A, a gene that codes for a diphtheria toxin A fragment.

or att $\mathrm{R}$ sequence, which is a prerequisite for LR recombination.) Likewise, the $3^{\prime}$ arm should be amplified using a forward primer with an att $\mathrm{B} 2$ sequence and a reverse primer with an at $\mathrm{B} 3$ sequence, and the amplified fragment must be subcloned into pDONR P2RP3. (In general, amplification of highly GC-rich regions should be avoided in these genomic PCRs, and PCR primers should not involve repetitive sequences such as Alu.) These processes, as well as the subsequent LR recombination reactions, can be quite easily performed according to the manufacturer's instructions. Additionally, owing to the absence of any ligation step and the presence of the MLS sequence in the vector backbone, it is unnecessary to search for appropriate restriction sites for vector construction/linearization. Moreover, as the BP and LR recombination reactions, unlike conventional ligation reactions, are highly efficient and specific, checking for approximately 10 Eschericheria coli transformants is sufficient to obtain correctly recombined plasmids in each step. Hence, with four primers harboring an appropriate $a t t \mathrm{~B}$ sequence (i.e., two primers for each arm), one can create targeting vectors with different positive selectable markers within 5-10 days (see Figures 1 and 3). In fact, we have constructed targeting vectors for more than 10 genes, within 1 week after primers' arrival.

Considerable efforts have been made in recent years to simplify targetingvector construction. For example, Kohli et al. (11) developed a facile method for generating recombinant adeno-associated virus-based targeting vectors using fusion PCR. Zhang et al. reported a simplified strategy for vector construction with the use of $E$. coli recombination cloning (12). Cottade-Almeida et al. (13) also utilized $E$. coli recombination cloning to generate mouse gene-targeting vectors. It should be noted, however, that these strategies still involve genomic library construction/screening, virus packaging, extensive restriction mapping, or DNA ligations. Gasperowicz et al. (14) recently reported a long-range PCR-based method that circumvented the need for unique restriction sites; however, this strategy still involved multiple DNA ligation steps. We would like to emphasize, therefore, that our strategy described above is simpler and quicker than any other methods reported thus far and involves none of their tedious or inefficient processes (i.e., library construction/screening, virus packaging, or searching for restriction sites for vector construction/ linearization).

\section{High-Efficiency Gene Targeting in a Human Cell Line}

To evaluate the usefulness of aboveconstructed targeting vectors for actual gene-targeting experiments, we used the Nalm- 6 cell line to examine the efficiency of gene targeting. We found that the gene-targeting efficiencies at the LIG4 (Figure 2) and KU80 (9) loci were $3.4 \%$ and $8.3 \%$, respectively. These results, together with previous reports by us and others (5-8), indicate that targeting vectors harboring positive and negative selectable markers are sufficient to disrupt virtually any genomic locus in Nalm-6 cells within 1 month. Previous work has suggested that the length of homologous DNA sequences (i.e., $5^{\prime}$ and $3^{\prime}$ arms) of the 


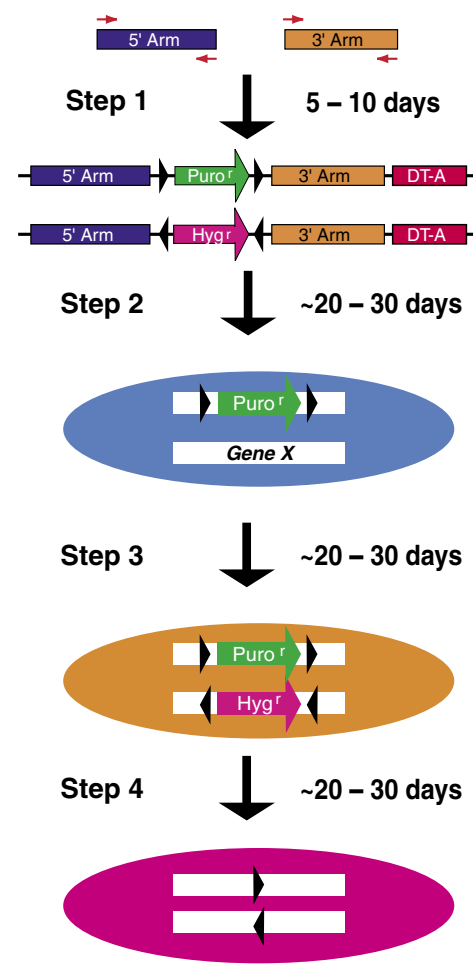

Figure 3. Strategy to generate human knockout cell lines. The strategy involves 4 steps: (Step 1) targeting-vector construction (see Figure 1); (Step 2) first gene targeting with a targeting vector; (Step 3) second gene targeting with another targeting vector; and (Step 4) Cre-mediated excision of drug-selectable markers. Step 4 is only required when additional gene(s) will be disrupted.

targeting vector is a major determinant of gene-targeting efficiencies (15-19), although recent work by $\mathrm{Lu}$ et al. (20) argues against this notion. Notably, in Nalm-6 cells, high-efficiency gene targeting is feasible with vectors with relatively short arms. Because short-arm vectors are sufficient for Nalm-6 cell gene targeting, drugresistant clones can be easily checked by genomic PCR, making screening processes simple and rapid. The PCR screening can be performed using a specific primer outside of a homologous arm region together with either of the two universal primers outside of the floxed markers (Figure 1). PCR-positive clones should then be subjected to Southern blot analysis to verify the correct targeting event.

In general, unlike the situation in animals, where homozygosity is achieved by breeding, both alleles must be disrupted to cause a loss-offunction phenotype for the study of autosomal genes in a cell. Thus, we performed targeted gene disruption on the second allele of the LIG4 gene (Figure 2), which allowed us to obtain homozygous mutant cells with the gene-targeting efficiency of $0.85 \%$. We therefore conclude that with the use of our system described here, homozygous knockout clones lacking a human gene of interest can be created within 2 to 3 months. In order to generate double mutant cells deficient for an additional gene(s), positive selectable markers (Hygr and Puror) must be removed from the $L I G 4$-null (LIG4Hyg/Puro) cells. As we reported previously (6), marker excision was easily performed with high efficiency (approximately 20\%) by transient expression of Cre recombinase, and the removal of both markers was verified by drug sensitivity as well as PCR and Southern blot analyses (data not shown). The outline of our strategy to generate human knockout cells is illustrated in Figure 3.

We have established a quick and simplified method for targeting vector construction. This method is readily applicable to gene-knockout experiments in ES cells as well as in virtually all sequenced organisms. We have also shown that Nalm-6 is highly proficient for gene-targeting experiments with thus created targeting vectors. Therefore, our system greatly facilitates gene-knockout experiments in human cells. The usefulness of Nalm-6 in gene targeting is quite intriguing and must be emphasized. Indeed, B-lymphocytes are reliable sources for analyzing gene functions via gene targeting, as exemplified by recent studies with the avian DT40 cell line. Additionally and more importantly, Nalm-6 is a human cell line that is karyotypically stable and expresses normal p53 (21). Hence, the results presented here underscore the usefulness of Nalm-6 in the postgenome era for reverse genetic studies of human genes.

\section{ACKNOWLEDGMENTS}

We thank Dr. Hirobumi Teraoka for generously providing us with the anti-LIG4 antibody. We also thank Dr. Noriyuki Sugo for helpful discussions and Kyoko Murai for excellent technical assistance. This work was supported in part by grants from Kato Memorial Bioscience Foundation (to N.A.) and Yokohama City University (Strategic Research Project no. W17005 to N.A.), and by Grant-in-Aids from the Ministry of Education, Culture, Sports, Science, and Technology (MEXT) of Japan (to N.A. and H.K.).

\section{COMPETING INTEREST STATEMENT}

The authors declare no competing interests.

\section{REFERENCES}

1. Capecchi, M.R. 1989. Altering the genome by homologous recombination. Science 244:1288-1292.

2. Vasquez, K.M., K. Marburger, Z. Intody, and J.H. Wilson. 2001. Manipulating the mammalian genome by homologous recombination. Proc. Natl. Acad. Sci. USA 98:84038410 .

3. Yamazoe, M., E. Sonoda, H. Hochegger, and S. Takeda. 2004. Reverse genetic studies of the DNA damage response in the chicken B lymphocyte line DT40. DNA Repair (Amst.) 3:1175-1185.

4. Yanez, R.J. and A.C. Porter. 1998. Therapeutic gene targeting. Gene Ther. 5:149159.

5. Grawunder, U., D. Zimmer, S. Fugmann, K. Schwarz, and M.R. Lieber. 1998. DNA ligase IV is essential for $\mathrm{V}(\mathrm{D}) \mathrm{J}$ recombination and DNA double-strand break repair in human precursor lymphocytes. Mol. Cell 2:477484.

6.So, S., N. Adachi, M.R. Lieber, and H. Koyama. 2004. Genetic interactions between BLM and DNA ligase IV in human cells. J. Biol. Chem. 279:55433-55442.

7.So, S., Y. Nomura, N. Adachi, Y. Kobayashi, T. Hori, Y. Kurihara, and H. Koyama. 2006. Enhanced gene targeting efficiency by siRNA that silences the expression of the Bloom syndrome gene in human cells. Genes Cells 11:363-371.

8. Adachi, N., S. So, S. Iiizumi, Y. Nomura, K. Murai, C. Yamakawa, K. Miyagawa, and H. Koyama. 2006. The human pre-B cell line Nalm-6 is highly proficient in gene targeting by homologous recombination. DNA Cell Biol. 25:19-24.

9. Uegaki, K., N. Adachi, S. So, S. Iiizumi, and H. Koyama. 2006. Heterozygous inactivation of human Ku70/Ku86 heterodimer does not affect cell growth, double-strand break repair, or genome integrity. DNA Repair (Amst.) 5:303-311.

10. Adachi, N., T. Ishino, Y. Ishii, S. Takeda, and H. Koyama. 2001. DNA ligase IV-deficient cells are more resistant to ionizing radiation in the absence of $\mathrm{Ku} 70$ : Implications for 
DNA double-strand break repair. Proc. Natl. Acad. Sci. USA 98:12109-12113.

11. Kohli, M., C. Rago, C. Lengauer, K.W. Kinzler, and B. Vogelstein. 2004. Facile methods for generating human somatic cell gene knockouts using recombinant adeno-associated viruses. Nucleic Acids Res. 32:e3.

12. Zhang, P., M.Z. Li, and S.J. Elledge. 2002. Towards genetic genome projects: genomic library screening and gene-targeting vector construction in a single step. Nat. Genet. 30:31-39

13. Cotta-de-Almeida, V., S. Schonhoff, T. Shibata, A. Leiter, and S.B. Snapper. 2003. A new method for rapidly generating gene-targeting vectors by engineering BACs through homologous recombination in bacteria. Genome Res. 13:2190-2194.

14. Gasperowicz, M., M. Kolanczyk, M. Stock, and F. Otto. 2004. Simplified method for gene targeting vector construction. BioTechniques 37:734-738.

15. Thomas, K.R. and M.R. Capecchi. 1987. Site-directed mutagenesis by gene targeting in mouse embryo-derived stem cells. Cell 51:503-512.

16. Shulman, M.J., L. Nissen, and C. Collins. 1990. Homologous recombination in hybridoma cells: dependence on time and fragment length. Mol. Cell. Biol. 10:4466-4472.

17. Hasty, P., J. Rivera-Perez, and A. Bradley. 1991. The length of homology required for gene targeting in embryonic stem cells. Mol. Cell. Biol. 11:5586-5591.

18. te Riele, H., E.R. Maandag, and A. Berns. 1992. Highly efficient gene targeting in embryonic stem cells through homologous recombination with isogenic DNA constructs. Proc. Natl. Acad. Sci. USA 89:5128-5132.

19. Deng, C. and M.R. Capecchi. 1992. Reexamination of gene targeting frequency as a function of the extent of homology between the targeting vector and the target locus. Mol. Cell. Biol. 12:3365-3371.

20. Lu, Z.H., J.T. Books, R.M. Kaufman, and T.J. Ley. 2003. Long targeting arms do not increase the efficiency of homologous recombination in the beta-globin locus of murine embryonic stem cells. Blood 102:1531-1533.

21. Filippini, G., S. Griffin, M. Uhr, H. Eppenberger, J. Bonilla, F. Cavalli, and G. Soldati. 1998. A novel flow cytometric method for the quantification of $\mathrm{p} 53$ gene expression. Cytometry 31:180-186.

Received 18 April 2006; accepted 7 June 2006.

Address correspondence to Noritaka Adachi or Hideki Koyama, Kihara Institute for Biological Research, Yokohama City University, Maioka-cho 641-12, Totsuka-ku, Yokohama 244-0813, Japan. e-mail:nadachi@yokohama-cu.ac.jp or koyama@yokohama-cu.ac.jp 